\title{
Blood pressure and anthropometrics of 4-y-old children born after preimplantation genetic screening: follow-up of a unique, moderately sized, randomized controlled trial
}

\author{
Jorien Seggers ${ }^{1}$, Maaike L. Haadsma², Sacha la Bastide-van Gemert ${ }^{3}$, Maas Jan Heineman4, Joke H. Kok ${ }^{5}$, Karin J. Middelburg ${ }^{4}$, \\ Tessa J. Roseboom ${ }^{4,6}$, Pamela Schendelaar ${ }^{1}$, Edwin R. Van den Heuvel ${ }^{3}$ and Mijna Hadders-Algra ${ }^{1}$
}

BACKGROUND: Recent studies suggest that in vitro fertilization (IVF) and intracytoplasmic sperm injection (ICSI) are associated with suboptimal cardiometabolic outcome in offspring. It is unknown whether preimplantation genetic screening (PGS), which involves embryo biopsy, affects blood pressure (BP), anthropometrics, and the frequency of received medical care. METHODS: In this prospective multicenter follow-up study, we assessed BP, anthropometrics, and received medical care of 4-y-old children born to women who were randomly assigned to IVF/ICSI with PGS $(n=49)$ or without PGS (controls; $n=64$ ). We applied linear and generalized linear mixed-effects models to investigate possible effects of PGS.

RESULTS: BP in the PGS and control groups was similar: 102/64 and 100/64 mm Hg, respectively. Main anthropometric outcomes in the PGS vs. control group were: BMI: 16.1 vs. 15.8; triceps skinfold: 108 vs. $98 \mathrm{~mm}$; and subscapular skinfold: 54 vs. $53 \mathrm{~mm}$ (all $P$ values > 0.05). More PGS children than controls had received paramedical care (speech, physical, or occupational therapy: 14 (29\%) vs. 9 (14\%); $P=0.03$ in multivariable analysis). The frequency of medicial treatment was comparable.

CONCLUSION: PGS does not seem to affect BP or anthropometrics in 4-y-old children. The higher frequency of received paramedical care after PGS may suggest an effect of PGS on subtle developmental parameters.

W orldwide, the number of couples undergoing in vitro fertilization (IVF), with or without intracytoplasmic sperm injection (ICSI), is gradually increasing (1). Nevertheless, pregnancy rates after IVF/ICSI are lower than desired. To enhance efficiency of IVF/ICSI, new techniques such as preimplantation genetic screening (PGS) have been developed.

In PGS, embryos are biopsied, and one or two blastomeres are aspirated and screened for aneuploidies. Only embryos with a normal chromosomal constitution are selected for transfer to the uterus (2). However, randomized controlled trials have indicated that PGS is not associated with higher pregnancy rates. As a matter of fact, reduced pregnancy rates after PGS are described for women of advanced maternal age $(3,4)$. As a consequence, PGS is no longer recommended.

Nevertheless, evaluation of the health of children born following PGS is relevant as it may reveal valuable information on long-term consequences of embryo biopsy. This is important as embryo biopsy is increasingly applied in the form of preimplantation genetic diagnosis (PGD). Little is known about the health outcome of children born following PGS. In this respect, it is noteworthy that recent reports indicated that IVF/ICSI by itself is associated with increased blood pressure (BP), altered body fat distribution, and vascular dysfunctions in offspring (5-9). Taking into account the general notion that the early environment shapes an individual's physical health later in life (10), it is conceivable that PGS, which includes more extensive embryo manipulation, introduces extra risks for adverse cardiometabolic outcome in offspring. Thus far, three cohort studies reported on child development in terms of anthropometrics and received medical care after PGS or PGD (11-13). The outcome of these cohort studies was reassuring, but anthropometrics and received medical care after PGS have never been reported using a randomized design. Furthermore, studies addressing cardiovascular health in PGS offspring are completely lacking.

The aim of the present study is to examine the effect of PGS on BP, anthropometrics, and received medical care of 4-y-old children. Our primary outcome measure was BP in $\mathrm{mm} \mathrm{Hg}$. Secondary outcomes were the presence of high BP, heart rate, pulse pressure, weight, standing height, BMI, the presence of obesity, triceps, and subscapular skinfold thickness, waist circumference, head circumference, and the frequency of hospitalization, consultation of a medical specialist, and of received paramedical treatment. The present study is part of

\footnotetext{
Division of Developmental Neurology, Department of Paediatrics, University of Groningen, University Medical Center Groningen, Groningen, The Netherlands; ${ }^{2}$ Division of Clinical Genetics, Department of Genetics, University of Groningen, University Medical Center Groningen, Groningen, The Netherlands; ${ }^{3}$ Department of Epidemiology, University of Groningen, University Medical Center Groningen, Groningen, The Netherlands; ${ }^{4}$ Department of Obstetrics and Gynaecology, Academic Medical Center, Amsterdam, The Netherlands; ${ }^{5}$ Department of Neonatology, Emma Children's Hospital, Amsterdam, The Netherlands; ${ }^{6}$ Department of Clinical Epidemiology, Biostatistics and Bioinformatics, Academic Medical Center, Amsterdam, The Netherlands. Correspondence: Mijna Hadders-Algra (m.hadders-algra@umcg.nl)
} 
a prospective, assessor-blinded, follow-up study of children born to women who were randomly assigned to IVF/ICSI with PGS or IVF/ICSI without PGS (controls) (4).

\section{RESULTS}

\section{Participation}

Between May 2003 and November 2005, 408 women were included in the randomized trial of which 52 women in the PGS group and 74 women in the control group had an ongoing pregnancy. Live birth of at least one child occurred in 49 and 71 women, respectively. Figure 1 shows eligibility, participation, and reasons for nonparticipation of children. Of the eligible couples, 40 couples in the PGS group (31 couples with singletons and 9 couples with a twin) and 53 couples in the control group (42 couples with singletons and 11 couples with a twin) participated in the follow-up assessment at $4 \mathrm{y}$. At child level, the overall postnatal attrition at the assessment at $4 \mathrm{y}$ was $18 \%$. Background variables for the children who were lost to follow-up were similar to those of children assessed at $4 \mathrm{y}$, with two exceptions: drop out in the control group was associated with lower parental education (Fisher's exact test; fathers: $P=0.040$; mothers: $P=0.006$ ), whereas drop out in the PGS group was associated with higher gestational age (Student's t-test; $P=0.040)$. We obtained BP measurements from $44 \mathrm{PGS}$ children ( $90 \%$ of included children) and 60 control children (94\%). Fourteen children partly cooperated and allowed one BP measurement instead of two (13\% of participating children). This single measurement was used in the analyses. Anthropometrics, perinatal characteristics, and parental characteristics did not differ between children who cooperated and those who did not cooperate in the BP measurement (data not shown).

\section{Infant and Parental Characteristics}

Parental, gestational, perinatal, and child characteristics are shown in Table 1. Most characteristics of the two groups were similar, but two differences were found. Gestational age was shorter $(38.7 \mathrm{wk})$ in the control group than that in the PGS group (39.4wk; $P=0.027$ ), and cesarean section was more often performed in the control group (in 38\%) than that in the PGS group (18\% of births; $P=0.039$ ).

\section{Stratification Singletons and Twins}

A different effect of PGS for singletons and twins could not be demonstrated for any of the outcome measures. The smallest $P$ value $(P=0.094)$ was observed for outcome measure heart rate. It was therefore decided that an effect of PGS can be further investigated for singletons and twins together.

\section{BP and Anthropometric Data}

Table 2 shows the results of the statistical analysis on the outcome measures for singletons and twins together. It indicates that none of the outcome measures were significantly affected by PGS when the possible correlation between twins is taken into account and the effect of PGS is corrected for center, and for assessor in case of numerical outcome measures. The sensitivity analysis did not change our conclusions, nor did it show that PGS had a different effect for IVF or ICSI children (data not shown).

A post hoc power analysis, based on a noncentral $t$-distribution, indicated that we were able to demonstrate a difference of $4.5 \mathrm{~mm} \mathrm{Hg}$ (or more) in the systolic BP (SBP) and of 4.0 $\mathrm{mm} \mathrm{Hg}$ (or more) in the diastolic BP (DBP) with the current sample size, using a power of $80 \%$ and a two-sided significance level of 0.05 . This means that the sample size of our study was

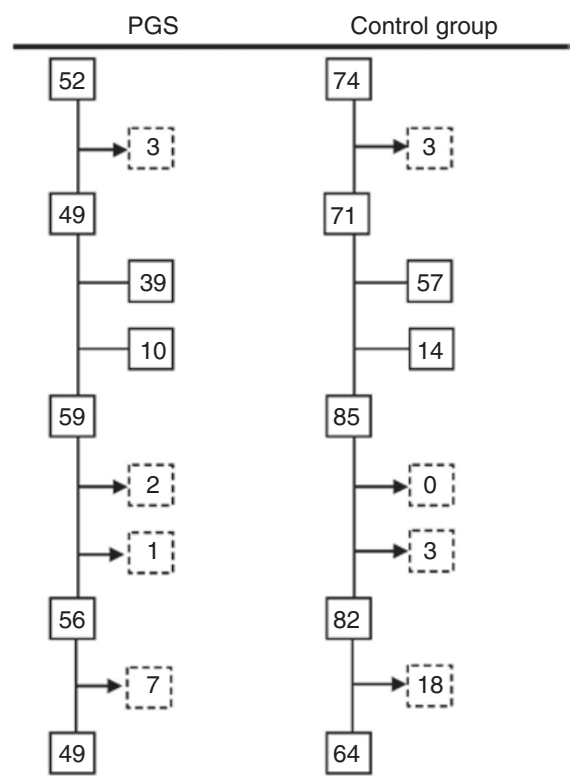

Figure 1. Eligibility and participation of children. aln the preimplantation genetic screening (PGS) group: one termination because of trisomy 18 , one intrauterine death due to placental abruption, and one immature birth. In the control group: one termination because of trisomy 18 , one termination because of a cleft lip and palate, and one intrauterine death. IIn the PGS group, a preterm delivery at $24 \mathrm{wk}+5 \mathrm{~d}$ resulted in the postpartum death of a twin. 'Couples who withdrew informed consent from the PGS trial were not approached for participation in the follow-up study. ${ }^{\mathrm{d}}$ Reasons for nonparticipation in the PGS group: 1 moving abroad, 6 assessment burden. In the control group: 5 moving abroad, 3 untraceable, and 10 assessment burden. 


\section{Articles | Seggerset al.}

Table 1. Infant and parental characteristics

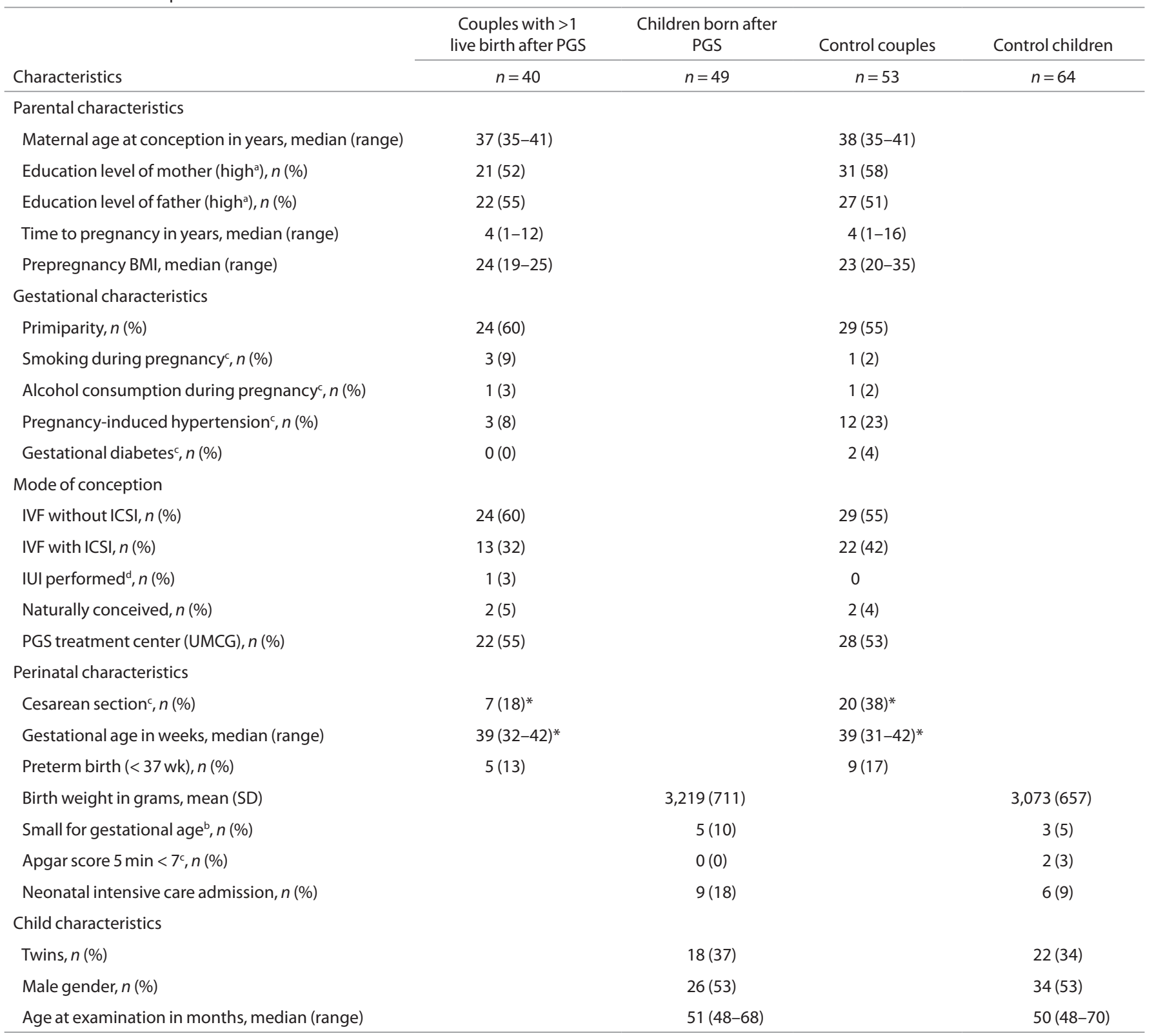

Mann-Whitney $U$ tests or Fisher's exact tests were used to compare between groups at parent level. At child level, linear or generalized linear mixed-effects models were applied to compare between groups in order to model possible correlation between twins.

ICSI, intracytoplasmic sperm injection; IUI, intrauterine insemination; IVF, in vitro fertilization; PGS, preimplantation genetic screening; UMCG, University Medical Center Groningen.

a University education or vocational colleges. ${ }^{b}$ Birth weight for gestational age is less than -2 SDs as compared with the Dutch reference population (Dutch reference tables). cMissing data in two groups: Apgar score 5 min $<7, n=8$; alcohol consumption during pregnancy, $n=12$; cesarean section, $n=1$; gestational diabetes, $n=5$; pregnancy-induced hypertension, $n=5$; smoking during pregnancy, $n=9$. ${ }^{d}$ In case of poor follicle growth, treatment was converted to intrauterine insemination. ${ }^{*} P<0.05$.

sufficiently large to detect relevant differences in our primary outcome measures (SBP and DBP) in case they would have been present.

Table 3 shows covariates that might be associated $(P<$ 0.250 ) with the outcome measures and the covariates that remained in the final model, after combining them in one statistical model and applying backward elimination at 5\% significance level. This more explorative analysis also showed that PGS has no statistically significant effect on BP or anthropometrics.

\section{Received Medical Care}

Received medical care in the two groups was largely comparable (Table 2). Hospitalization had occurred in 18 PGS children (37\%) vs. 18 control children (28\%), and 25 PGS children $(51 \%)$ vs. 28 control children (44\%) had been referred to a medical specialist. Univariate analyses showed a trend for a higher frequency of received paramedical treatment in PGS children as compared with controls (Table 2; 14 PGS children $(29 \%)$ vs. 9 control children (14\%); $P=0.124)$. Again, the sensitivity analysis did not alter our conclusions. The explorative 
Table 2. Blood pressure, anthropometrics, and received medical care

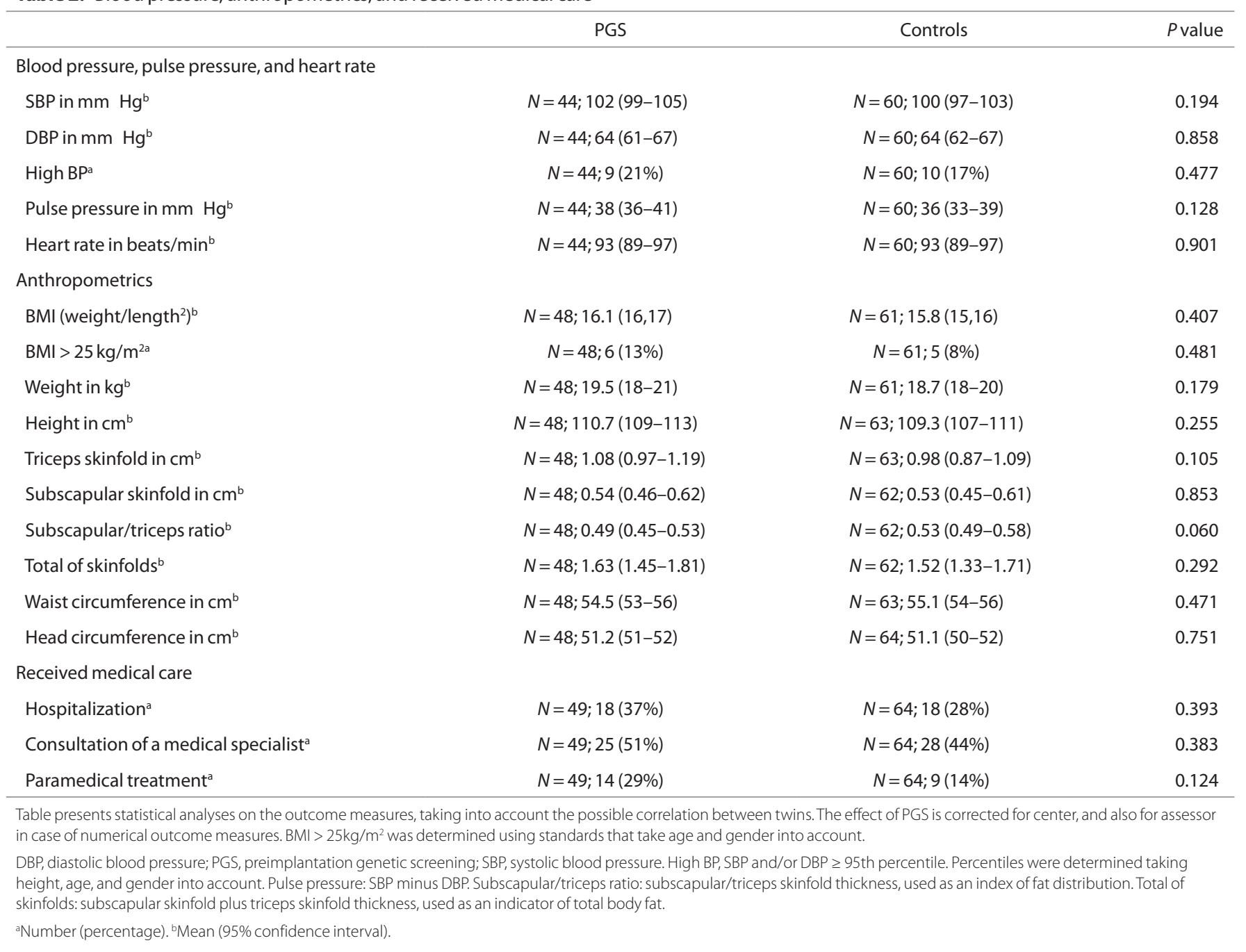

multivariable analyses however showed that PGS was significantly associated with a higher frequency of paramedical treatment (Table 3; $P=0.032$ ).

No differences between the two groups were observed in the frequency or type of medication prescribed, nor in the occurrence of febrile seizures, epilepsy, or cerebral palsy. Medication had been prescribed to 23 PGS (47\%) children and to 29 control children (45\%), antibiotics had been the most frequently prescribed drugs (PGS: $n=15$ (31\%); controls: $n=13(20 \%)$ ). In the PGS group, 2 children (4\%) had one or more febrile seizures as compared with 2 control children (3\%). None of the children were diagnosed with epilepsy. One PGS child was diagnosed with cerebral palsy.

The number and indications for surgical interventions were comparable in the two groups. In the PGS group, 10 operative procedures were performed in 7 children (14\%); in the control group, 14 procedures in 10 children (16\%). Adenotonsillectomy was the most frequently performed procedure (2 PGS (4\%) and 6 control (9\%) children). Three PGS children (6\%) underwent orchidopexy and 1 control child (2\%) had correction of penile hypospadia.

\section{DISCUSSION}

The present study did not demonstrate statistically significant, nor clinically relevant, differences in BP or anthropometrics between children conceived with IVF/ICSI with PGS or IVF/ ICSI without PGS. To our knowledge, BP levels after PGS have not been reported before. In addition, our study found a higher frequency of received paramedical treatment in PGS children as compared with controls. Other parameters of received medical care were comparable between the two groups.

Our findings on anthropometrics and received medical care are comparable with those published by others: growth parameters after embryo biopsy applied in PGD/PGS are within the normal range (11-13), and the frequency of hospital admission and surgical interventions is not increased after PGD/PGS (11-16). The explorative analyses of our study suggested that PGS children more often received paramedical treatment than controls. However, this finding could be the result of selection bias; drop out in the control group was associated with lower educational levels of the parents, and drop out in the PGS group was associated with higher gestational age of the children. In addition, more children were lost to follow-up in the control 


\section{Articles | Seggerset al.}

Table 3. Explorative analyses: covariates potentially associated with outcome parameters

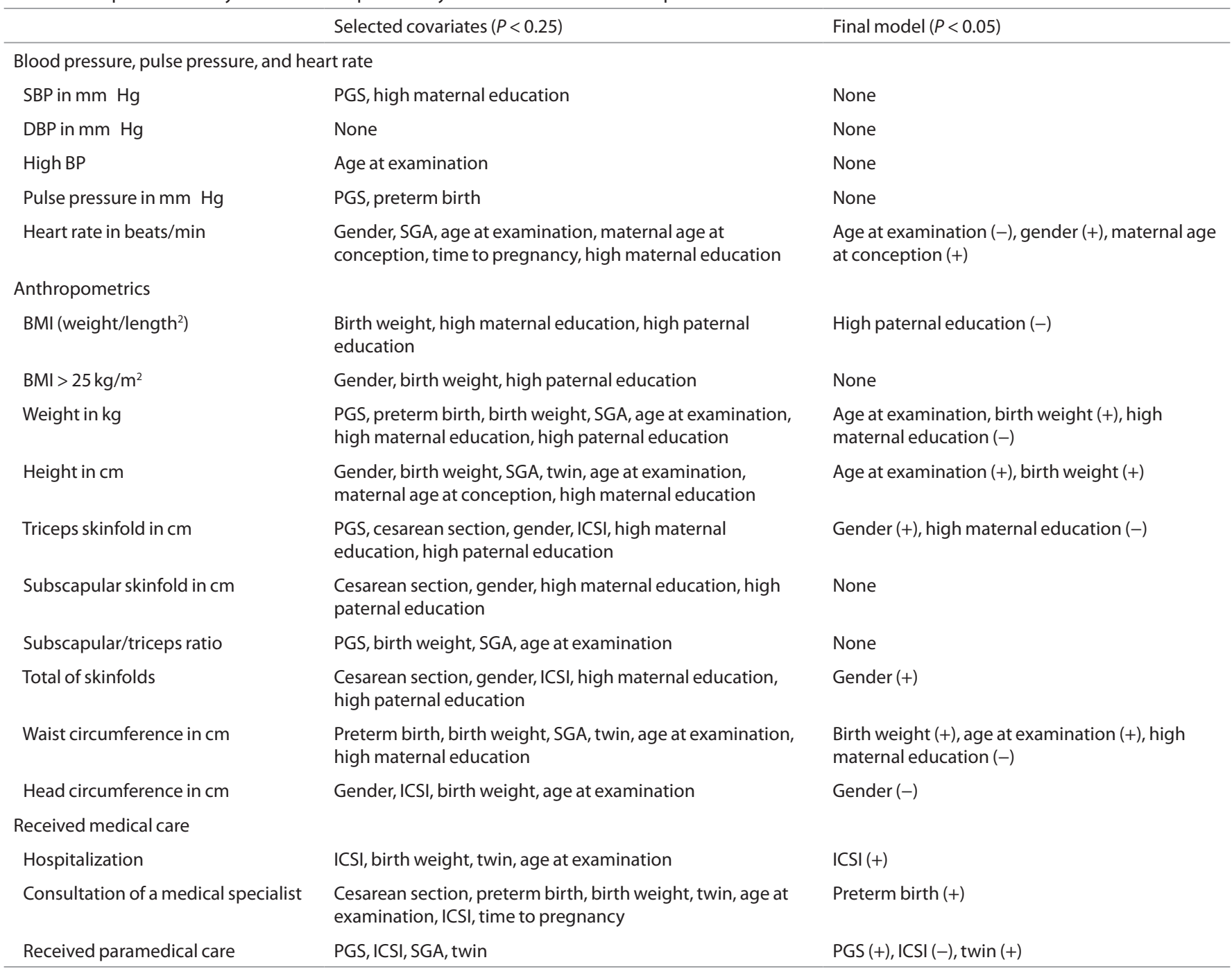

Table shows the covariates that might be associated $(P<0.25)$ with the outcome measures and the covariates that remained in the final model, after combining them in one statistical model and applying backward elimination at significance level $5 \%$. PGS was only significantly associated with an increased prevalence of received paramedical care $(P=0.032)$. In the final model: the plus sign indicates that the outcome increases with a positive change in the covariate; for a dichotomous outcome measure, the plus sign indicates that the probability of the event increases. A plus sign for gender means that girls have higher outcome measures.

BP, blood pressure; DBP, diastolic blood pressure; ICSI, intracytoplasmic sperm injection; PGS, preimplantation genetic screening; SBP, systolic blood pressure; SGA, small for gestational age. High BP, SBP and/or DBP $\geq 95$ th percentile. Percentiles were determined taking height, age, and gender into account. Pulse pressure: SBP minus DBP.

group than that in the PGS group (22\% of eligible children in the control group vs. $13 \%$ in the PGS group). As parents were aware of group allocation (information provided $>12 \mathrm{wk}$ of pregnancy), it could be that couples in the PGS group were more worried about the development of their child and therefore continued to participate in the study. The same worried attitude, together with the selection bias, may have induced an increased frequency of received paramedical treatment. On the other hand, the higher frequency of paramedical treatment may be a sign of suboptimal neurodevelopment after PGS, as was suggested by the study of Middelburg et al. (17).

The plausible association of PGS and adverse developmental outcome is based on the "developmental origins of health and disease hypothesis," which states that adaptive responses to environmental stimuli during critical developmental periods in early life may have long-term consequences due to reprogramming of metabolic and endocrine key systems (10). For example, manipulations in maternal diet during pregnancy are associated with high BP in the offspring of a number of mammalian species $(18,19)$, and in humans prenatally exposed to the Dutch famine, coronary artery disease is more often reported. Notably, the adverse effect of exposure to famine was independent of birth weight (20). IVF and ICSI are also suggested to change the early environment in such a manner that it may have long-term consequences for the offspring (21). Indeed, several studies suggested subtle cardiometabolic alterations in IVF/ICSI offspring $(5,6,8,9)$. This was partly expected, as IVF and ICSI conceptions more often result in preterm birth and low birth weight, which are risk factors for adiposity and hypertension (22). However, the cardiometabolic alterations in the children of the aforementioned studies could not be attributed to preterm birth or low birth weight 
alone. Various aspects of IVF that might compromise the environment of the early embryo are: the use of ovarian hyperstimulation, which results in artificial maturation of follicles and an altered uterine environment, and the use of culture media in which the first cell divisions take place (23). Embryo biopsy is an invasive procedure that in theory could induce damage to the embryo. It is conceivable that embryo biopsy in addition to the IVF/ICSI procedure induces more changes to the early environment, which can potentially negatively influence longterm cardiometabolic status. This is especially true as embryo biopsy was performed at the cleavage stage, when all cells are totipotent, meaning that cellular differentiation at that point is absent (24). In theory, removing one of these cells may affect the growth rate of the embryo or affect health outcome of children. Yet, the present study suggests that PGS does not add to putative adverse risks of IVF.

\section{Strengths and Weaknesses}

To our knowledge, this is the first study investigating BP levels after embryo biopsy. A strength of our study is its design. We studied children born to women who were randomly assigned to IVF/ICSI with PGS or IVF/ICSI without PGS, resulting in comparable background variables between the two groups. Other strengths are the prospective and assessor-blinded study design. As couples were invited before pregnancy, we reduced the chance of selection bias based on the child's health, and participation rates were high. Furthermore, it is the only study evaluating anthropometrics and received medical care in children born following PGS alone, instead of in a combined group of children born following PGS and PGD. The technical procedures of PGD are similar to those of PGS, but the indication for PGD is to screen for and select embryos against specific genetic diseases in high-risk couples (25). This means that couples undergoing PGD are often fertile, whereas couples undergoing PGS are subfertile. Subfertility is known to be associated with more obstetrical problems, perinatal problems, and a higher rate of congenital abnormalities (26-28). It is also associated with an increased risk of cardiovascular disease later in life of the subfertile women themselves (29). Children born to subfertile PGS couples could for these reasons be more at risk for adverse health outcome than children born following PGD. It is therefore reassuring that even in this relatively vulnerable population, embryo biopsy did not seem to increase the risk of adverse cardiometabolic outcome. Furthermore, the randomized design of the present study resulted in a well-matched control group. This design cannot be applied in studies focusing on development after PGD, as randomization of couples at risk for a child with a genetic disorder is unethical.

In the present study, both singletons and twins were included, which contributes to the generalizability of the results. However, it could be argued that twins and singletons should not be analyzed together, as twins are more often born preterm or with a low birth weight, which are risk factors for adiposity and hypertension (22). Nevertheless, our first analysis indicated that PGS had no different effect on all of our outcome measures for singletons and twins, and we took into account the possible correlation structure for twins in all analyses. Furthermore, the study also included IVF and ICSI children. As it is unknown whether IVF and ICSI share the same cardiometabolic risk for offspring (30), we performed a sensitivity analysis. We found no significant differences in outcome measures for IVF and ICSI children. Another limitation is that the study was not designed to detect differences in BP but to detect differences in ongoing pregnancy rates (4). However, the post hoc power analysis indicated that we were able to demonstrate a difference of $4.5 \mathrm{~mm} \mathrm{Hg}$ in the SBP and of $4.0 \mathrm{~mm} \mathrm{Hg}$ in the DBP, in case these differences would have been present. This means that the power of our study is sufficient to detect clinically relevant differences in our primary outcome measures (SBP and DBP). However, as this is the first study concluding that embryo biopsy has no consequences for BP levels, the finding should be confirmed by other studies. In addition, our study may not be powered for all secondary outcome measures. Therefore, other studies with preferably larger sample sizes should also confirm the absence of other adverse effects of PGS. For this purpose, our data could also be used in future meta-analyses, which may help overcome sample-size limitations of the current follow-up studies.

Another limitation is the number of $\mathrm{BP}$ measurements performed. It is well known that several BP measurements are necessary to reveal actual BP. Due to logistical reasons, we measured BP twice on $1 \mathrm{~d}$, instead of on several separate occasions. We used the mean of the two measurements for the analysis. In case the 4-y-old children did not cooperate and allowed only one BP measurement, this single measurement was used for the analysis. The difficulty of obtaining multiple follow-up measurements is also acknowledged by other studies in the field. The price of multiple assessments is a substantially higher attrition rate. Therefore, studies evaluating the effects of reproductive technologies all opted to measure $\mathrm{BP}$ on a single day $(7,9,30)$. For the follow-up at older ages, a 24 -h telemetric approach could be a good alternative. As assessments took place in the University Medical Center Groningen (UMCG) or at home, it could be hypothesized that the location of assessment influenced BP levels. However, as an equal number of PGS and control children were assessed at home, and as we corrected for center in the analyses, it is unlikely that this would change the results.

It should also be noted that newer techniques are being developed in order to enhance pregnancy rates and that these techniques may allow for faster and more reliable results. In the present study, embryos were biopsied at the cleavage stage, in which the embryo consists of four to eight cells. It is hypothesized that PGS as performed in the current study does not increase pregnancy rates due to embryo mosaicism at day 3 , and because only 8 chromosomes were tested for aneuploidies instead of 22 pairs of autosomes and 1 pair of sex chromosomes. Newer techniques in which the trophectoderm (precursor of the placenta) is biopsied at the blastocyst stage ( $\sim 5 \mathrm{~d}$ after fertilization), instead of the inner cell mass at the cleavage stage ( $\sim 3 \mathrm{~d}$ after fertilization), may decrease the likelihood of mosaicism $(24,25)$. The effects of prolonged embryo culture 
are however not well known (23), but recently, Mäkinen et al. (31) suggested that blastocyst culture is linked to an increase in the proportion of large for gestational age infants. Properly designed randomized controlled trials are needed to evaluate the safety and efficiency of new techniques before they are implemented to the clinical setting (32).

In conclusion, the results of the present study indicate that PGS, a procedure involving embryo biopsy, does not seem to negatively influence BP or anthropometrics of 4-y-old children. However, we did find an increased consumption of paramedical care in the PGS group. Whether this is the result of selection bias, a chance finding, or whether it indicates that PGS may affect more subtle parameters of developmental outcome is uncertain. Even though PGS is no longer practiced on routine basis, sufficiently powered studies should address this question as embryo biopsy is still performed in PGD and in new techniques that are being developed to improve pregnancy rates $(33,34)$.

\section{METHODS}

\section{Recruitment}

Participants were the children born to women who participated in a double-blind, multicenter, randomized controlled trial on the efficiency of PGS on improving ongoing pregnancy rates (ISRCTN76355836). The inclusion criteria for the trial were as follows: female aged 35-41 y, no preceding failed IVF or ICSI, and no objections against a possible double-embryo transfer. Randomization was performed centrally before the in vitro procedure with minimization for female age (35-37 or 38-41 y), the application of ICSI, and with stratification for study center (UMCG, Groningen, The Netherlands, or Academic Medical Center, Amsterdam, The Netherlands). Between May 2003 and November 2005, a total of 408 women were randomly assigned to undergo IVF with or without PGS. Treatment was continued until June 2006.

\section{PGS Procedure}

Embryos of women assigned to PGS underwent laser-assisted biopsy (Hamilton Thorne Biosciences, Beverly, MA) of a single blastomere in case the embryo contained at least four blastomeres, $3 \mathrm{~d}$ after follicular aspiration. A second blastomere was only biopsied in case no nucleus suitable for fluorescence in situ hybridization was available and only if the remaining embryo contained at least four blastomeres. Fluorescence in situ hybridization analysis was performed for 8 chromosomes: chromosomes 1,16 , and 17 were analyzed with chromosome enumeration probes (Vysis, Downers Grove, IL) on the day of the biopsy, and chromosomes 13, 18, 21, X, and Y were analyzed the next day with MultiVysion PGT probes (Vysis). Embryos were categorized as normal in case the fluorescence in situ hybridization analysis showed two copies of each autosome and XX or XY, and as abnormal in case a different chromosomal constitution was found, and as undetermined if no determination of chromosomal constitution could be made, due to a failed biopsy, the absence of a nucleus in the blastomere, an incomplete nucleus, or failure of the fluorescence in situ hybridization procedure. Embryos containing fewer than four blastomeres were not biopsied and were also categorized as undetermined. The two chromosomally normal embryos with the most optimal morphologic features (based on the number and regularity of blastomeres and the percentage of fragmentation) were transferred to the uterus on day 4. Undetermined embryos with good morphologic features were transferred in case no chromosomally normal embryos with good morphological features were available for transfer. Chromosomally abnormal embryos were never transferred. Embryos of women not assigned to PGS were selected solely on the basis of morphologic features (also based on the number and regularity of blastomeres and the percentage of fragmentation).
Before randomization, couples provided written informed consent for the PGS trial and in case of a successful pregnancy, also for the follow-up protocol. When an ongoing pregnancy was achieved (>12 wk), couples were informed about their allocation. At each follow-up assessment, parental consent for participation of their child(ren) in the study was reconfirmed. The study design was approved by the respective medical ethics committees of both the hospitals and by the Dutch Central Committee of Research Involving Human Subjects. Additional details on treatment procedures and study design can be found in the study by Mastenbroek et al. (4).

\section{Follow-Up Examination}

Information on demographics and the prenatal and perinatal period were recorded on standardized charts at the first follow-up assessment at the age of $2 \mathrm{wk}$. Medical records provided information on fertility diagnosis and time to pregnancy.

When the children were $4 \mathrm{y}$ of age, the follow-up assessment included measurement of BP and an assessment of anthropometrics by trained researchers who were blinded to mode of conception. Assessments were carried out between October 2009 and November 2010 in the UMCG or at home. Parents who received IVF/ICSI (with or without PGS) in the UMCG were invited for assessment at the Institute of Developmental Neurology, UMCG. Children of parents who received treatment in the Academic Medical Center were assessed at home due to logistical reasons. We used an automated BP monitor, the Datascope Accutorr plus (Datascope, Mahwah, NJ), with an appropriate cuff size to measure BP and heart rate at the nondominant arm while the child was seated. Two measurements were performed. The first measurement took place after a cognitive test, and the second measurement after collection of the anthropometric data, $\sim 5$ min after the first BP measurement. The mean of the two readings was used to record SBP, DBP, pulse pressure, and heart rate. To extract a clinically relevant elevated BP from the crude measurements, percentiles that take age, gender, and height into account were used: SBP and/or DBP $\geq 95$ th percentile denoted the presence of "high BP" (35). Body weight was recorded to the nearest $0.1 \mathrm{~kg}$ using an electronic scale (Radwag, Radom, Poland). Standing height was measured to the nearest $0.1 \mathrm{~cm}$ with a stadiometer (Seca, Hamburg, Germany). BMI was calculated with the formula: weight/height ${ }^{2}$ and was subsequently classified into normal or obese using standards that take gender and age into account (36). Triceps and subscapular skinfold thickness was measured three times, alternating between the two skinfolds, using a Harpenden caliper. The mean of the three measurements was used as outcome parameter. The triceps skinfold was used as a measure of peripheral fat, the subscapular skinfold as a measure for truncal fat, the sum of subscapular and triceps skinfold as an indicator of total body fat, and the subscapular/triceps skinfold ratio was used as an index of fat distribution. Waist and occipitofrontal head circumference were measured with a nonstretchable "lasso" tape in millimeters. All measurements were performed while the child was wearing underwear only.

The parents filled out a questionnaire on received medical care from birth to the assessment at the age of $4 \mathrm{y}$. Data collection included information on surgical interventions, hospital admission, consultations of a medical specialist (general practitioner excluded), use of paramedical care (speech therapy, physical therapy, or occupational therapy), febrile seizures, epilepsy, and the use of medical drugs.

\section{Sample Size}

The sample size of the study was based on the primary outcome of the original PGS trial: the number of women needed to detect an increase in ongoing pregnancy rates (4). In order to estimate the number of children needed per group to detect a relevant difference in SBP and DBP, knowledge on the SD in BP levels for 4-y-old Dutch children is required. Exact data are however not available, but the following estimates can be provided: for an SD of $\sim 6 \mathrm{~mm} \mathrm{Hg}, 24$ children per group are needed to detect a difference of $\geq 5 \mathrm{~mm} \mathrm{Hg}$ with $80 \%$ power at the two-sided significance level of 0.05 , using a two-sample $t$-test. For an SD of 8 or $10 \mathrm{~mm} \mathrm{Hg}$, the required sample size increases to 42 or 64 children per group, respectively. On the basis of these sample size calculations, it is expected that our study is sufficiently large, but 
a post hoc power analysis for BP levels will be conducted to investigate this afterwards.

\section{Statistical Analysis}

Parental, subfertility, and perinatal characteristics were compared between the two groups using Mann-Whitney $U$-tests, Fisher's exact tests, and linear or generalized linear mixed-effects models, when applicable.

A linear or a generalized linear mixed-effects model was applied to investigate a possible effect of PGS on the outcome measures. The cluster variable was the mother of the children to model possible correlation between twins. At first, we tested whether the effect of PGS was different for singletons and twins. In case of a significant difference, the effect of PGS was determined for both the groups separately, and if no difference was detected, the effect of PGS was determined for all children together to maximize power.

For the numerical outcome measures (i.e., SBP, DBP, pulse pressure, heart rate, height, weight, BMI, triceps skinfold thickness, subscapular skinfold thickness, skinfold ratio, total skinfold thickness, waist circumference, and head circumference) a linear mixed-effects model was used, corrected for center and assessor and with a random intercept for mother. The Satterthwaite approach for the number of degrees of freedom for the type III tests of PGS were applied.

For the binary outcome measures (i.e., high BP, BMI $>25 \mathrm{~kg} / \mathrm{m}^{2}$, hospitalization, referral to a medical specialist, and received paramedical treatment), generalized estimating equations and the logit link function were used together with the robust or empirical estimator and an exchangeable working correlation matrix. The effects of PGS were corrected for center, and the type III generalized score statistics was applied to determine the $P$ values.

These analyses were performed to the intention-to-treat principle. They were repeated without children born to couples who conceived spontaneously $(n=4)$ or via intrauterine insemination $(n=1)$. This sensitivity analysis also included an investigation of an interaction effect of ICSI or IVF with PGS.

For an explorative analysis, we first investigated which covariates affected the outcome measures individually at a significance level of $\alpha=$ 0.25 . The following covariates were included: ICSI, time to pregnancy, maternal and paternal age at conception, maternal and paternal level of education, maternal prepregnancy BMI, smoking during pregnancy, use of alcohol during pregnancy, pregnancy-induced hypertension, gestational diabetes, cesarean section, gender, twin status, gestational age, preterm birth, birth weight, small for gestational age, and age at examination. Subsequently, the selected covariates were combined in one statistical model for that specific outcome measure, and backward elimination of the covariates was applied at a significance level of $\alpha=$ 0.05 . For the numerical outcome measures, all analyses were corrected for center and assessors, and for the dichotomous response variables, all analyses were corrected for center only. The statistical technique for the explorative analysis used the same linear or generalized linear mixed-effects model with mother as the cluster variable.

Analyses were performed with Predictive Analytics SoftWare version 18.0 (IBM, Armonk, NY) and Statistical Analysis System version 9.2 (SAS Institute, Cary, NC). In all analyses, $P$ values $<0.05$ were considered statistically significant.

\section{ACKNOWLEDGMENTS}

We thank the parents and children who participated in the study; Marjolein Jongbloed-Pereboom, Rosan Aapkes, Elise Bennik, Hanneke Geut, and Bertine de Vries for their help with the assessments; and Linze Dijkstra, Michiel Schrier, and Loes de Weerd for their technical assistance.

\section{STATEMENT OF FINANCIAL SUPPORT}

The randomized controlled trial was funded by a grant (945-03-013) from the Netherlands Organization for Health Research and Development (ZonMw). The follow-up at 4 years was financially supported by the University Medical Center Groningen, the Cornelia Foundation, the Junior Scientific Master Class, and the Research School of Behavioural and Cognitive Neurosciences.

Disclosure: The authors declare no conflict of interest.

\section{REFERENCES}

1. de Mouzon J, Goossens V, Bhattacharya S, et al.; European IVF-monitoring (EIM) Consortium, for the European Society of Human Reproduction and Embryology (ESHRE). Assisted reproductive technology in Europe, 2006: results generated from European registers by ESHRE. Hum Reprod 2010;25:1851-62.

2. Wilton L. Preimplantation genetic diagnosis for aneuploidy screening in early human embryos: a review. Prenat Diagn 2002;22:512-8.

3. Hardarson T, Hanson C, Lundin K, et al. Preimplantation genetic screening in women of advanced maternal age caused a decrease in clinical pregnancy rate: a randomized controlled trial. Hum Reprod 2008;23:2806-12.

4. Mastenbroek S, Twisk M, van Echten-Arends J, et al. In vitro fertilization with preimplantation genetic screening. N Engl J Med 2007;357:9-17.

5. Belva F, Painter R, Bonduelle M, Roelants M, Devroey P, De Schepper J. Are ICSI adolescents at risk for increased adiposity? Hum Reprod 2012;27:257-64.

6. Ceelen M, van Weissenbruch MM, Roos JC, Vermeiden JP, van Leeuwen FE, Delemarre-van de Waal HA. Body composition in children and adolescents born after in vitro fertilization or spontaneous conception. J Clin Endocrinol Metab 2007;92:3417-23.

7. Ceelen M, van Weissenbruch MM, Vermeiden JP, van Leeuwen FE, Delemarre-van de Waal HA. Cardiometabolic differences in children born after in vitro fertilization: follow-up study. J Clin Endocrinol Metab 2008;93:1682-8.

8. Scherrer U, Rimoldi SF, Rexhaj E, et al. Systemic and pulmonary vascular dysfunction in children conceived by assisted reproductive technologies. Circulation 2012;125:1890-6.

9. Sakka SD, Loutradis D, Kanaka-Gantenbein C, et al. Absence of insulin resistance and low-grade inflammation despite early metabolic syndrome manifestations in children born after in vitro fertilization. Fertil Steril 2010;94:1693-9.

10. Barker DJ. Fetal origins of coronary heart disease. BMJ 1995;311:171-4.

11. Banerjee I, Shevlin M, Taranissi M, et al. Health of children conceived after preimplantation genetic diagnosis: a preliminary outcome study. Reprod Biomed Online 2008;16:376-81.

12. Desmyttere S, Bonduelle M, Nekkebroeck J, Roelants M, Liebaers I, De Schepper J. Growth and health outcome of 102 2-year-old children conceived after preimplantation genetic diagnosis or screening. Early Hum Dev 2009;85:755-9.

13. Desmyttere S, De Schepper J, Nekkebroeck J, et al. Two-year auxological and medical outcome of singletons born after embryo biopsy applied in preimplantation genetic diagnosis or preimplantation genetic screening. Hum Reprod 2009;24:470-6.

14. Strom CM, Levin R, Strom S, Masciangelo C, Kuliev A, Verlinsky Y. Neonatal outcome of preimplantation genetic diagnosis by polar body removal: the first 109 infants. Pediatrics 2000;106:650-3.

15. Liebaers I, Desmyttere S, Verpoest W, et al. Report on a consecutive series of 581 children born after blastomere biopsy for preimplantation genetic diagnosis. Hum Reprod 2010;25:275-82.

16. European Society of Human Rreproduction and Embryology Preimplantation Genetic Diagnosis Consortium Committee. Preimplantation genetic diagnosis consortium. Hum Reprod 2007;22:232-36.

17. Middelburg KJ, van der Heide M, Houtzager B, et al.; PGS Follow-up Study Group. Mental, psychomotor, neurologic, and behavioral outcomes of 2 -year-old children born after preimplantation genetic screening: followup of a randomized controlled trial. Fertil Steril 2011;96:165-9.

18. Gopalakrishnan GS, Gardner DS, Rhind SM, et al. Programming of adult cardiovascular function after early maternal undernutrition in sheep. Am J Physiol Regul Integr Comp Physiol 2004;287:R12-20.

19. Kwong WY, Wild AE, Roberts P, Willis AC, Fleming TP. Maternal undernutrition during the preimplantation period of rat development causes blastocyst abnormalities and programming of postnatal hypertension. Development 2000;127:4195-202.

20. Roseboom TJ, van der Meulen JH, Osmond C, et al. Coronary heart disease after prenatal exposure to the Dutch famine, 1944-45. Heart 2000;84:595-8.

21. Watkins AJ, Fleming TP. Blastocyst environment and its influence on offspring cardiovascular health: the heart of the matter. J Anat 2009;215:52-9. 


\section{Articles | Seggers et al.}

22. Rogers I; EURO-BLCS Study Group. The influence of birthweight and intrauterine environment on adiposity and fat distribution in later life. Int J Obes Relat Metab Disord 2003;27:755-77.

23. Pinborg A, Wennerholm UB, Romundstad LB, et al. Why do singletons conceived after assisted reproduction technology have adverse perinatal outcome? Systematic review and meta-analysis. Hum Reprod Update 2013;19:87-104.

24. Brezina PR, Brezina DS, Kearns WG. Preimplantation genetic testing. BMJ 2012;345:e5908.

25. Ly KD, Agarwal A, Nagy ZP. Preimplantation genetic screening: does it help or hinder IVF treatment and what is the role of the embryo? J Assist Reprod Genet 2011;28:833-49.

26. Baird DD, Wilcox AJ, Kramer MS. Why might infertile couples have problem pregnancies? Lancet 1999;353:1724-5.

27. Draper ES, Kurinczuk JJ, Abrams KR, Clarke M. Assessment of separate contributions to perinatal mortality of infertility history and treatment: a case-control analysis. Lancet 1999;353:1746-9.

28. Zhu JL, Basso O, Obel C, Bille C, Olsen J. Infertility, infertility treatment, and congenital malformations: Danish national birth cohort. BMJ 2006;333:679.

29. Parikh NI, Cnattingius S, Mittleman MA, Ludvigsson JF, Ingelsson E. Subfertility and risk of later life maternal cardiovascular disease. Hum Reprod 2012;27:568-75.
30. Belva F, Roelants M, De Schepper J, et al. Blood pressure in ICSI-conceived adolescents. Hum Reprod 2012;27:3100-8.

31. Mäkinen S, Söderström-Anttila V, Vainio J, Suikkari AM, Tuuri T. Does long in vitro culture promote large for gestational age babies? Hum Reprod 2013;28:828-34.

32. Harper J, Magli MC, Lundin K, Barratt CL, Brison D. When and how should new technology be introduced into the IVF laboratory? Hum Reprod 2012;27:303-13.

33. Harper J, Coonen E, De Rycke M, et al. What next for preimplantation genetic screening (PGS)? A position statement from the ESHRE PGD Consortium Steering Committee. Hum Reprod 2010;25: $821-3$.

34. Geraedts J, Collins J, Gianaroli L, et al. What next for preimplantation genetic screening? A polar body approach! Hum Reprod 2010;25: 575-7.

35. National High Blood Pressure Education Program Working Group on High Blood Pressure in Children and Adolescents. The fourth report on the diagnosis, evaluation, and treatment of high blood pressure in children and adolescents. Pediatrics 2004;114:555-76.

36. Cole TJ, Bellizzi MC, Flegal KM, Dietz WH. Establishing a standard definition for child overweight and obesity worldwide: international survey. BMJ 2000;320:1240-3. 